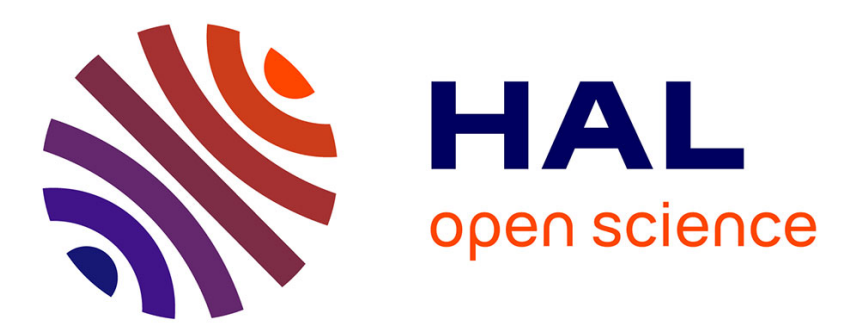

\title{
ZONES MORTES DANS LES CHAMBRES PROPORTIONNELLES A CATHODES GRAPHITÉES
}

\author{
G. Remy, G. Schultz, B. Stringfellow
}

\section{To cite this version:}

G. Remy, G. Schultz, B. Stringfellow. ZONES MORTES DANS LES CHAMBRES PROPORTIONNELLES A CATHODES GRAPHITÉES. Congres de la Societe Francaise de Physique, 1977, Poitiers, France. pp.C3-33-C3-37, 10.1051/jphyscol:1978306 . jpa-00217410

\section{HAL Id: jpa-00217410 https://hal.science/jpa-00217410}

Submitted on 1 Jan 1978

HAL is a multi-disciplinary open access archive for the deposit and dissemination of scientific research documents, whether they are published or not. The documents may come from teaching and research institutions in France or abroad, or from public or private research centers.
L'archive ouverte pluridisciplinaire HAL, est destinée au dépôt et à la diffusion de documents scientifiques de niveau recherche, publiés ou non, émanant des établissements d'enseignement et de recherche français ou étrangers, des laboratoires publics ou privés. 


\title{
ZONES MORTES DANS LES CHAMBRES PROPORTIONNELLES A CATHODES GRAPHITÉES
}

\author{
G. REMY, G. SCHULTZ (*) et B. STRINGFELLOW \\ Centre de Recherches Nucléaires, (Laboratoire PNPP/HE), Université Louis Pasteur, \\ 67037 Strasbourg Cedex, France
}

\begin{abstract}
Résumé. - En vue de la réalisation d'un détecteur cylindrique (pour la détection de toutes les particules chargées émises à partir d'un vertex d'interaction sur des faisceaux très intenses de particules), une chambre test proportionnelle multifils à cathodes pleines (graphitées) présentant une zone de faible amplification a été réalisée.

Une étude systématique de l'efficacité de détection de la chambre et du facteur d'amplification a été effectuée au voisinage de la zone morte en fonction de certains critères électroniques et géométriques. Quelques conclusions intéressantes permettant d'obtenir un fonctionnement optimum de la chambre sont dégagées.
\end{abstract}

\begin{abstract}
A small flat proportional chamber with continuous (graphite deposited) cathodes incorporating a region of small amplification has been constructed. The method will eventually be used in a vertex detector consisting of cylindrical MWPC in a magnetic field and capable of operating in intense incident beams.

A systematic study of the efficiency of the chamber and the amplification factor has been made in the neighbourhood of this dead spot as a function of various electronic and geometric criteria. The results of these measurements are presented here.
\end{abstract}

1. Introduction. - Un détecteur de vertex comportant de 5 à 7 chambres proportionnelles multifils cylindriques est en développement au laboratoire PNPP/HE du CRN de Strasbourg. Ce détecteur installé dans le champ magnétique d'un aimant doit être placé dans un faisceau très intense émanant de l'accélérateur du Super Synchrotron à Protons du C.E.R.N. (environ $10^{7}$ particules par impulsion de faisceau et par $\mathrm{cm}^{2}$ ) ; il est donc nécessaire de rendre inefficaces les chambres aux endroits de traversée du faisceau principal, et ceci pour deux raisons essentielles :

1. pour ne pas détériorer prématurément la chambre. Pour un mélange gazeux d'argon-isobutane contenant quelques pour cent de méthylal, la durée de vie d'une chambre est environ de $2 \times 10^{11}$ particules $/ \mathrm{cm}^{2}\left({ }^{1}\right)$, soit dans notre cas à peine deux jours ;

2. pour éviter par suite de la saturation des fils touchés de confondre dans une même coïncidence de déclenchement un bon événement avec d'autres particules du faisceau.

Quelques travaux [1-3] ont été publiẹs sur la façon d'obtenir des zones mortes dans les chambres proportionnelles. Généralement, les solutions envisa-

(*) Adresse actuelle : Laboratoire d'Électronique et d'Instrumentation Nucléaire, Université du Haut-Rhin, Mulhouse, France.

(1) Sauli, F., Communication privée. gées sont irréversibles et une zone morte ne peut plus être réactivée $[1,2]$. Dans notre cas, plusieurs conditions doivent être satisfaites :

1. Les zones mortes doivent être compatibles avec le projet du détecteur de vertex qui présente des cathodes pleines (graphitées) de masse volumique faible.

2. Il doit être facile de rendre efficace à la détection une zone morte afin d'étudier pendant l'expérience la configuration du faisceau, vérifier à faible intensité le bon fonctionnement de la zone morte, etc.

3. La position des zones mortes de la chambre doit pouvoir être modifiée si on change l'énergie des particules incidentes ou la valeur de l'induction magnétique de l'aimant. Ce problème est lié au point précédent et conduit à envisager une structure en damiers.

Avant d'équiper le détecteur cylindrique de zones mortes, nous avons construit une chambre test plane et étudié systématiquement ses propriétés d'amplification et d'efficacité de détection au voisinage d'une zone morte. Les résultats préliminaires des mesures sont donnés dans cet article.

2. Banc et chambre de test. Caractéristiques générales. - La chambre de test présente une distance intercathode de $12 \mathrm{~mm}$ et une distance de $2 \mathrm{~mm}$ entre les fils d'anode. Les cathodes sont réalisées à l'aide d'un dépôt de graphite d'un côté de feuilles de 
mylar de $50 \mu \mathrm{m}$ d'épaisseur tendues sur des cadres circulaires d'aluminium. La zone morte est confectionnée en même temps que la cathode. Des bagues d'acier carrées de $35,1 \mathrm{~mm}$ de côté et de différentes largeurs ( $2 \mathrm{~mm}, 3 \mathrm{~mm}$ ou $4 \mathrm{~mm}$ ) jouent le rôle de cache lors du dépôt de graphite et déterminent la zone comprise entre le dead spot et la cathode. Cette zone exempte de toute poussière de graphite doit demeurer parfaitement isolante.

Pour réaliser les contacts électriques sur la zone morte et la cathode, nous nous sommes inspirés d'une technique développée au Laboratoire de l'Accélérateur Linéaire d'Orsay ( $\left.{ }^{2}\right)$. Le contact se fait de la même façon sur la zone morte et la cathode. La haute tension est amenée à l'aide d'un fil fin $(\varnothing=0,2 \mathrm{~mm})$ entouré d'une gaine de téflon (fil à wrapper). L'extrémité de ce fil qui doit réaliser le contact avec la cathode (ou la zone morte) est d'abord soudée sur une fine pastille d'aluminium de $5 \mathrm{~mm}$ de diamètre et $10 \mu \mathrm{m}$ d'épaisseur.

Une perforation à l'aide d'une aiguille $(\varnothing \approx 0,3$ à $0,5 \mathrm{~mm}$ ) est ménagée en périphérie de la cathode. L'aiguille est introduite à partir de la face graphitée de manière à éviter la présence d'une protubérance dans l'espace interélectrode.

La pastille d'aluminium est ensuite encollée à l'aide d'une colle à prise rapide puis appliquée centralement sur la perforation du côté du mylar non graphité.

Le contact électrique entre le graphite d'un côté et la pastille d'aluminium de l'autre est assuré par l'application d'une goutte de laque d'argent sur l'orifice apparent dans la face graphitée (Fig. 1).

La chambre est alors montée de façon classique où les plans de cathode sont portés à la haute tension (négatives) et les fils de mesure à la masse.

Les mesures ont été réalisées à l'aide d'une source d'électrons de ${ }^{90} \mathrm{Sr}$ collimée $(\sigma \simeq 0,7 \mathrm{~mm})$. Le déclenchement de la chambre est assuré par deux scintillateurs en coïncidence. La chambre est montée sur une vis micrométrique, les scintillateurs et la source étant fixes. Les fils d'anode sont équipés par une électronique classique (préamplificateur, amplificateur, discriminateur). La figure 2 donne une vue d'ensemble de cet équipement de test.

Le mélange gazeux utilisé comporte $79 \%$ d'argon, $17 \%$ d'isobutane et $4 \%$ de méthylal.

Le plateau d'efficacité de détection est représenté sur la figure 3 pour différentes tensions seuil des discriminateurs. La tension anode-cathode $V_{\mathrm{AC}}$ de $3150 \mathrm{~V}$ a été retenue comme point de fonctionnement. L'efficacité de détection de la chambre est liée au seuil de déclenchement des discriminateurs ainsi qu'aux performances des préamplificateurs et des amplificateurs. Aussi est-il impératif de ramener cette tension seuil au nombre de charges collectées

(2) Lefrançois, J. et Delcros, P., Communication privée.

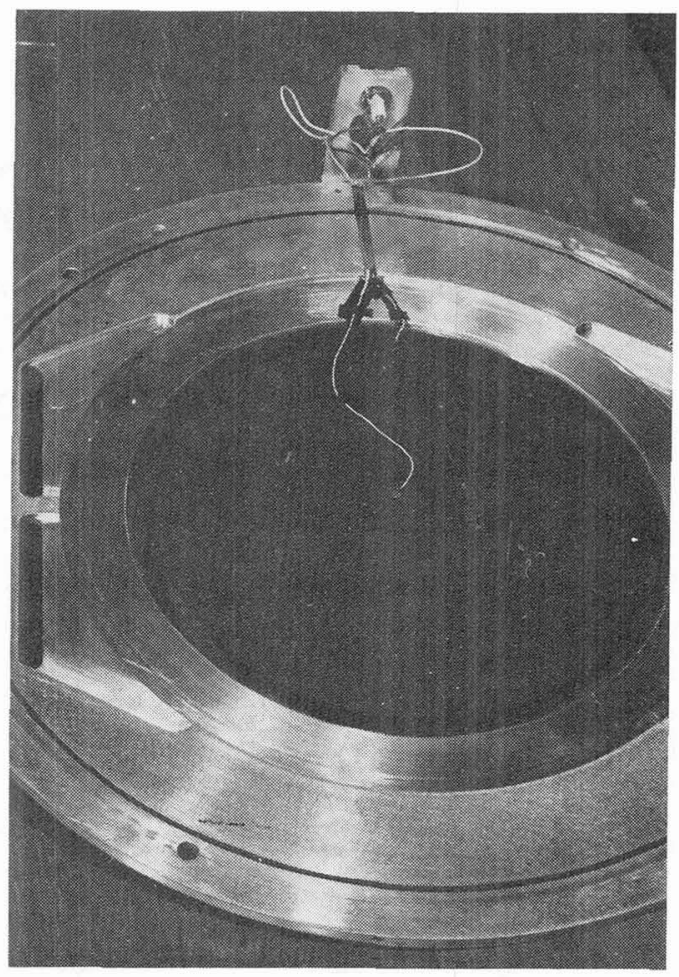

FIG. 1. - Détail des contacts électriques réalisés sur la zone morte et sur la cathode.

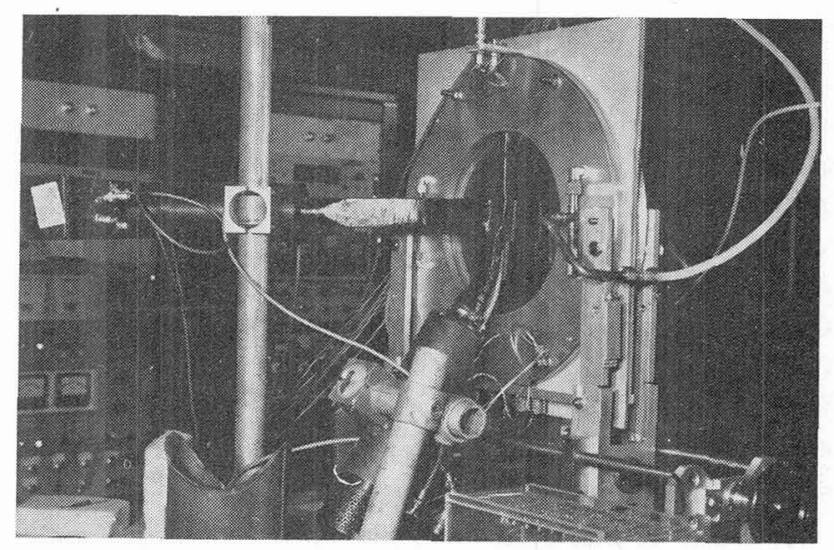

FIG. 2. - Vue d'ensemble de l'équipement de mesure.

sur l'anode afin de conférer aux mesures un caractère général. Cette correspondance tensions de déclenchement des discriminateurs-nombre de charges collectées sur l'anode est montrée sur la figure 4. Généralement, le seuil de déclenchement des discriminateurs est de $50 \mathrm{mV}$ ce qui correspond à environ $3 \times 10^{5}$ charges collectées sur l'anode. La variation du facteur de multiplication $M$ de la chambre en fonction de la tension d'anode est représentée à la figure 5 . Pour obtenir le nombre total de charges collectées sur l'anode, $M$ doit être multiplié par le nombre total $n$ d'électrons formés au passage des particules ionisantes (électrons d'une source ${ }^{90} \mathrm{Sr}$ ) soit environ en moyenne 135 pour notre gaz et pour 


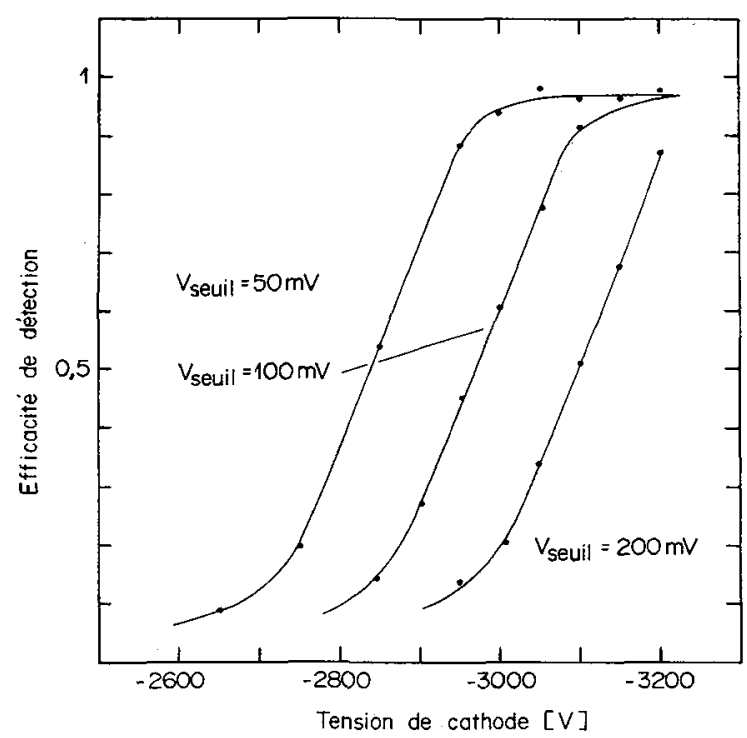

FrG. 3. - Plateau d'efficacité de détection pour différentes tensions seuil des discriminateurs. La chambre présente une distance intercathode de $12 \mathrm{~mm}$. Le pas des fils d'anode est de $2 \mathrm{~mm}$. Le gaz utilisé est $79 \%$ d'argon, $17 \%$ d'isobutane et $4 \%$ de méthylal. Le point de fonctionnement retenu est $V_{\text {AC }}=3150 \mathrm{~V}$.

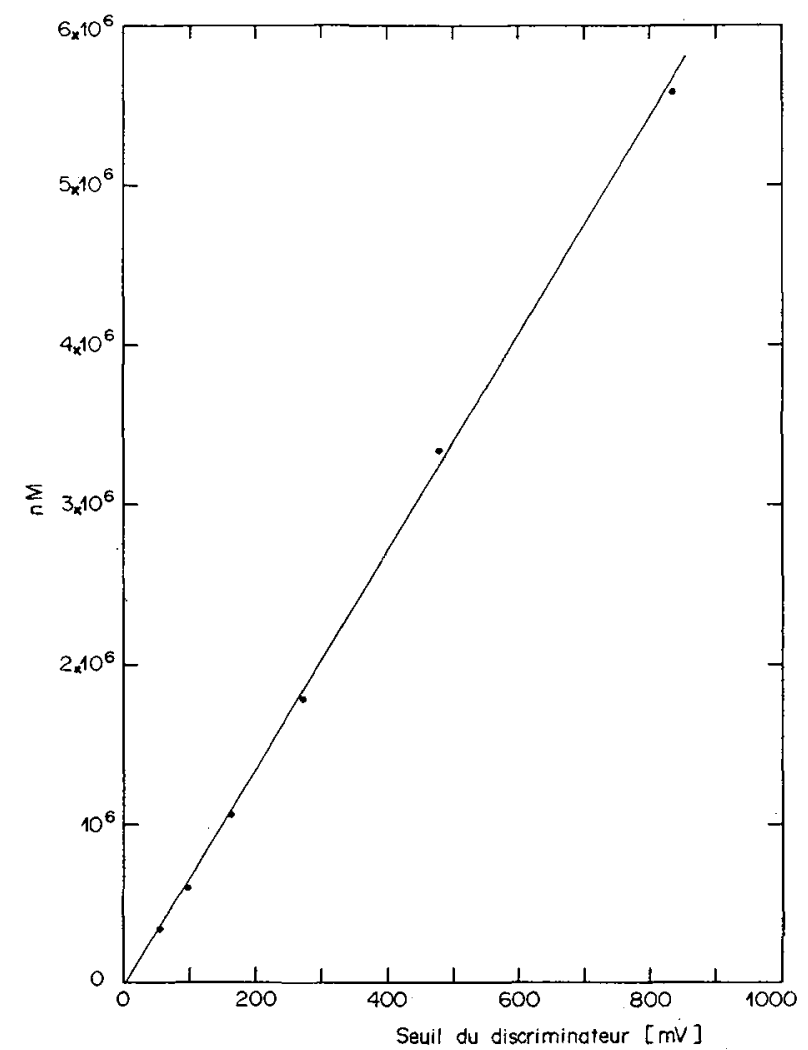

FIG. 4. - Correspondance tensions seuil des discriminateurscharges collectées sur l'anode.

une distance intercathode de $12 \mathrm{~mm}$ [4]. Pour notre point de fonctionnement $\left(V_{\mathrm{AC}}=3150 \mathrm{~V}\right), n M$ est environ égal à $4 \times 10^{6}$ en moyenne soit 13 fois plus élevé que le seuil de déclenchement normal des discriminateurs $(50 \mathrm{mV})$, valeur tout à fait normale et couramment utilisée.

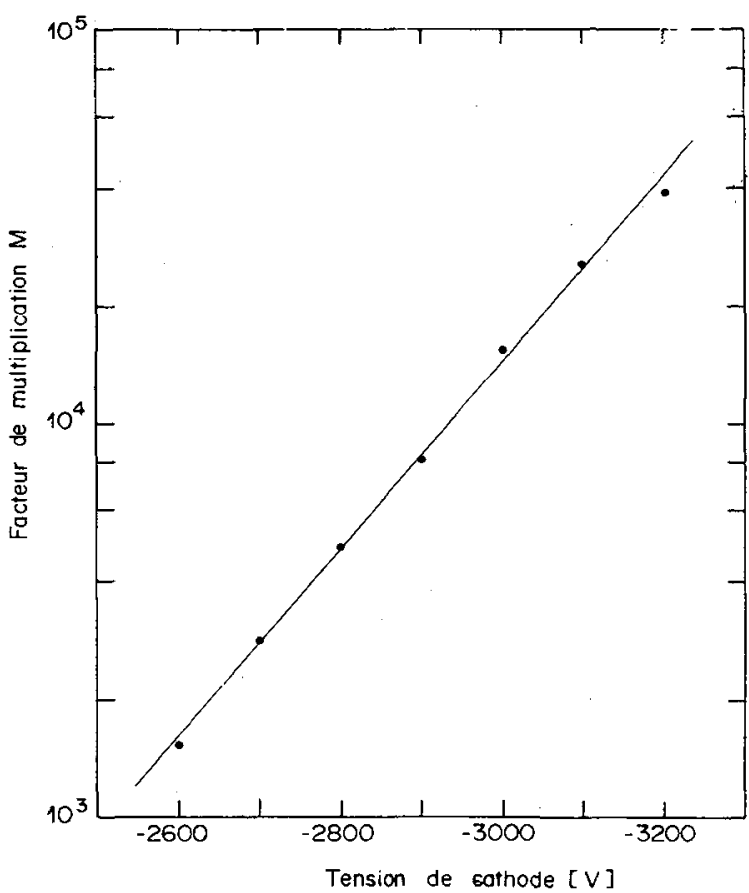

Fig. 5. - Variation du facteur de multiplication $M$ de la chambre en fonction de la tension anode-cathode $V_{\mathrm{AC}}$.

3. Mesures d'efficacité. - L'efficacité de détection qui a été mesurée au milieu de la zone aveugle, est fonction de la différence de potentiel $\Delta V$ appliquée entre cette zone et la cathode. L'efficacité de détection diminue pour atteindre une valeur minimum d'environ 0,001 pour $\Delta V$ supérieur à $800 \mathrm{~V}$ (Fig. 6). Nous avons constaté que cette valeur correspond au taux de coïncidences fortuites.

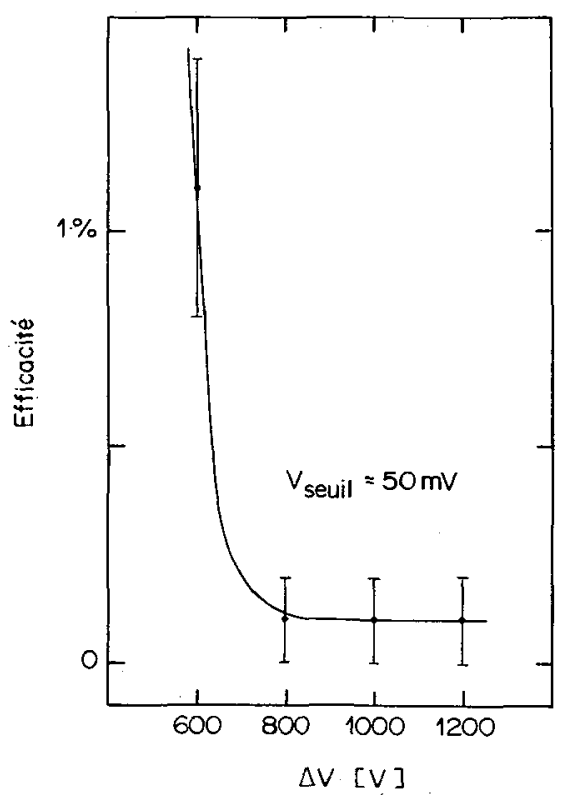

FIG. 6. - Efficacité de détection au milieu de la zone morte en fonction de la variation de potentiel $\Delta V$ entre la zone morte et la cathode. Pour $\Delta V>800 \mathrm{~V}$, l'efficacité de détection devient inférieure à $1 / 1000$. 
Une première mesure d'efficacité locale de détection a été réalisée pour une zone morte carrée présentant des coins arrondis dont la distance entre la zone morte et la cathode est de $2 \mathrm{~mm}$. Comme la collimation du faisceau d'électrons de la source de ${ }^{90} \mathrm{Sr}$ présente une dispersion supérieure au pas de $2 \mathrm{~mm}$ des fils d'anode, les signaux des différents fils d'anode ont été sommés. La courbe d'efficacité locale est montrée à la figure 7 .

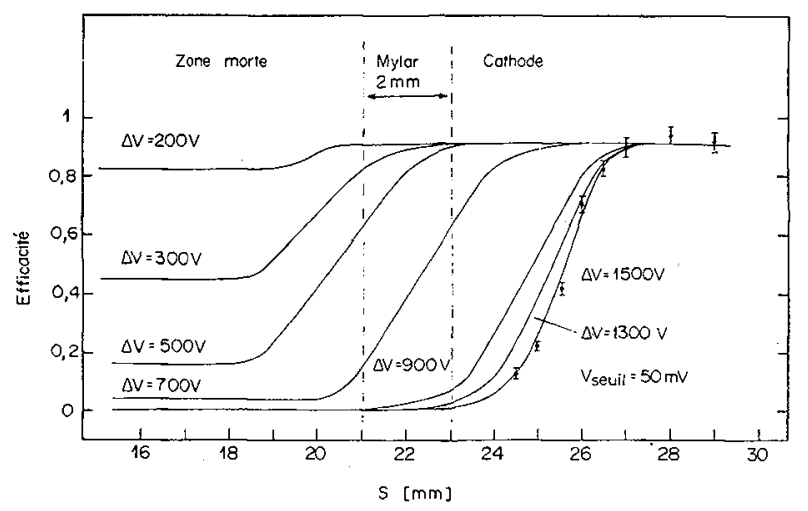

FrG. 7. - Efficacité locale de détection pour une distance : zone morte-cathode $d$ égale à $2 \mathrm{~mm}$. On constate que les courbes tendent vers une position de saturation quand $\Delta V$ augmente. La zone morte réelle est d'environ $4 \mathrm{~mm}$ plus grande que la zone morte physique découpée dans la cathode.

Outre le fait qu'une efficacité de détection inférieure à 0,001 est atteinte pour $\Delta V>.800 \mathrm{~V}$ (voir aussi la Fig. 6), on constate que les courbes tendent vers une position de saturation quand $\Delta V$ augmente. De plus, la zone morte réelle est environ $4 \mathrm{~mm}$ plus grande que la zone morte physique découpée dans la cathode.

Les mêmes mesures ont été alors répétées pour une zone morte carrée à coins arrondis présentant une distance entre la zone morte et la cathode de 3 et $4 \mathrm{~mm}$. Pour ces mesures, la source collimée est placée juste en face des fils d'anode et la chambre est déplacée avec un pas égal à celui des fils d'anode soit $2 \mathrm{~mm}$. La mesure est effectuée sur le fil placé en face du collimateur. Dans ce cas, la pleine efficacité de détection correspond à environ $85 \%$ (dispersion du faisceau d'électrons collimés de la source de ${ }^{90} \mathrm{Sr}$ supérieure au pas de $2 \mathrm{~mm}$ des fils de mesure).

Le résultat de ces mesures est présenté à la figure 8, simultanément pour un espace de séparation entre zone morte et cathode de $3 \mathrm{~mm}$ et de $4 \mathrm{~mm}$. On peut en déduire les mêmes conclusions que précédemment; cependant pour un espace de séparation de $4 \mathrm{~mm}$ la saturation dans la position des courbes est moins nette et la taille réelle de la zone morte augmente encore $(6 \mathrm{~mm}$ contre $4 \mathrm{~mm}$ précédemment).

Nous avons également effectué des mesures d'efficacité locale avec zone morte et cathode au

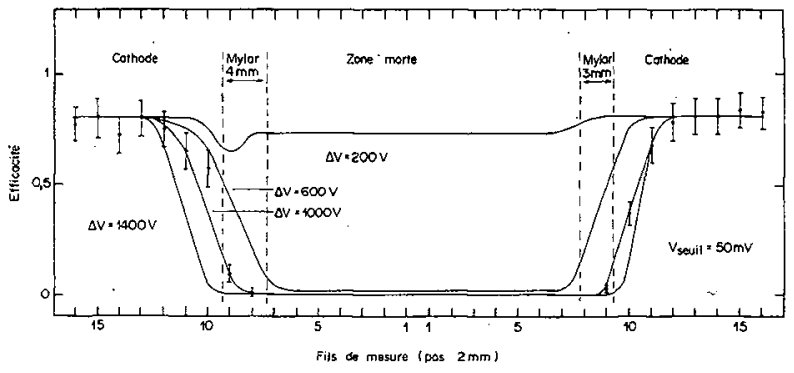

FIG. 8. - Mêmes résultats que ceux montrés sur la figure 7 mais pour $d=3 \mathrm{~mm}$ et $d=4 \mathrm{~mm}$.

même potentiel $\left(V_{\mathrm{AC}}=3150 \mathrm{~V}\right)$. Le résultat de ces mesures est présenté à la figure 9 pour 3 valeurs de tension seuil des discriminateurs (voir la Fig. 4). Même pour $V_{\text {seuil }}=50 \mathrm{mV}$, il apparaît un creux d'inefficacité pour une séparation zone mortecathode de $4 \mathrm{~mm}$. Le creux n'apparaît pas pour une séparation de $3 \mathrm{~mm}$ jusqu'à un seuil de déclenchement de $100 \mathrm{mV}$ bien que les signaux d'anode soient atténués pour les fils au voisinage immédiat de la zone de séparation.

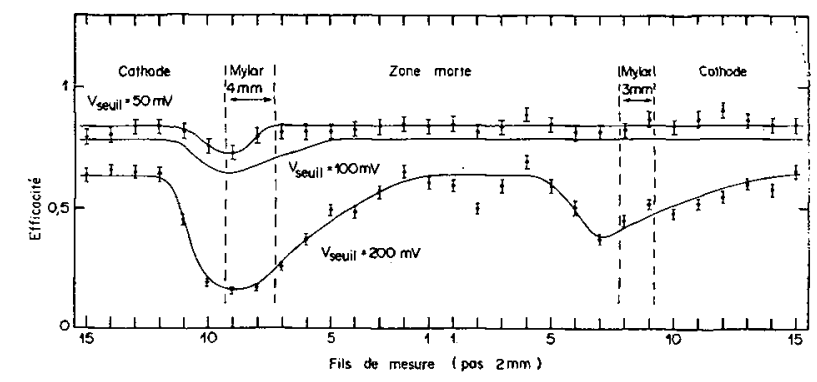

FIG. 9. - Efficacité locale de détection relevée avec un potentiel identique sur la zone morte et sur la cathode $(3150 \mathrm{~V})$ pour $d=3 \mathrm{~mm}$ et $d=4 \mathrm{~mm}$. Même pour une tension seuil des discriminateurs égale à $50 \mathrm{mV}$, il apparaît un creux d'inefficacité pour $d=4 \mathrm{~mm}$.

4. Conclusion. - De ces mesures, pour la construction des zones mortes du détecteur de vertex, nous avons retenu une distance de séparation entre zone morte et cathode de $3 \mathrm{~mm}$. Cette distance devrait permettre des différences de potentiel $\Delta V$ entre zone morte et cathode importantes sans risque de claquage. De plus, il n'apparaît pas de creux d'inefficacité dans la zone de séparation pour des valeurs normales de seuil de déclenchement de l'ensemble pré-amplificateur-amplificateur discriminateur lié aux fils de mesure.

Le point de fonctionnement retenu est $V_{\mathrm{AC}}=3150 \mathrm{~V}$ avec $\Delta V=1000 \mathrm{~V}$. Dans ce cas l'efficacité de détection est inférieure à $1 / 1000$ dans la zone morte. En se reportant à la figure 5 , on constate que $\Delta V=1000 \mathrm{~V}$ fait diminuer le coeffi- 
cient de multiplication $M$ d'environ un facteur 240 et amène la durée de vie de la chambre à environ 400 jours. Si ceci n'est pas suffisant, $\Delta V$ doit être augmenté.

Il est prévu de tester une nouvelle chambre comportant une zone morte de $5 \times 14 \mathrm{~mm}^{2}$ et une distance de séparation entre zone morte et cathode de $3 \mathrm{~mm}$. Dans ce cas, la surface de la zone de séparation est plus grande que celle de la zone morte. Si les mesures sont concluantes, une cham- bre à damiers sera réalisée et des tests à haute intensité effectués.

Remerciements. - Un groupe de physiciens de 1'Accélérateur Linéaire d'Orsay et du « Lézard » du C.E.R.N. ont développé des chambres proportionnelles à zones mortes et les ont réalisées d'une façon quasi identique. Nous remercions Monsieur Lefrançois du L.A.L. pour les différentes discussions et conseils que nous avons reçus à propos de ce travail.

\section{Bibliographie}

[1] ADERHolz, M., Lazeyras, P., Lehraus, I., MatTeWson, R et TEJESSY, W., Nucl. Instrum. Methods 123 (1975) 237.

[2] CRITtenden, R. R. et KRIDER, J. C., Nucl. Instrum. Methods 128 (1975) 599.
[3] MAJEWSKI, S. et SAULI, F., C.E.R.N. NP Internal Report 75-14 (7 Nov. 1975).

[4] SChultZ, G., Thèse soutenue à 1'Université Louis Pasteur de Strasbourg le 8-7-1977 (№ d'ordre : 1015). 